\title{
Single-mode performance analysis for vertical-cavity surface-emitting
}

\section{lasers}

\section{Journal Article}

\section{Author(s):}

Odermatt, Stefan; Witzigmann, Bernd; Eitel, Sven

Publication date:

2007

Permanent link:

https://doi.org/10.3929/ethz-b-000007902

\section{Rights / license:}

In Copyright - Non-Commercial Use Permitted

\section{Originally published in:}

Journal of Computational Electronics 6(1-3), https://doi.org/10.1007/s10825-006-0093-7 


\title{
Single-mode performance analysis for vertical-cavity surface-emitting lasers
}

\author{
Stefan Odermatt · Bernd Witzigmann · Sven Eitel
}

Published online: 18 January 2007

(C) Springer Science + Business Media, LLC 2007

\begin{abstract}
In this work, the simulation of the single-mode stability in vertical-cavity surface-emitting lasers (VCSELs) is presented using a microscopic electro-opto-thermal model. Experimental data for oxide-confined VCSELs emitting at $850 \mathrm{~nm}$ with different contact metal designs are also available. It is shown that detailed models for the optical losses in the cavity consisting of outcoupling and absorption are required in order to explain the experiments. The role of cavity losses and spatial hole burning in the nonlinear electro-optothermal simulation framework is discussed in a quantitative manner.
\end{abstract}

Keywords VCSEL $\cdot$ TCAD $\cdot$ Simulation $\cdot$ Single-mode stability

\section{Introduction}

Vertical-cavity surface-emitting lasers (VCSELs) are fundamental components in current and future communication and sensing systems, since they offer many advantages at low cost like on-wafer testing, high quantum efficiency and welldefined single-mode behavior. Comprehensive simulators are becoming essential tools to explore the parameter space for an optimum design solution and they allow to get deeper insight into the internal device physics.

In this work, the single-mode performance of $850 \mathrm{~nm}$ oxide-confined VCSEL devices using multidimensional

S. Odermatt $\cdot$ B. Witzigmann $(\bowtie)$

Integrated Systems Laboratory, ETH Zurich,

CH-8092 Zürich, Switzerland

e-mail: bernd@iis.ee.ethz.ch

S. Eitel

Avalon Photonics Ltd., CH-8048 Zürich, Switzerland electro-opto-thermal simulation models [1] is analyzed. The influence of the contact metallization design on the maximum single-mode power is investigated in detail. The qualitative and quantitative agreement of simulation and measurement results for different contact designs illustrates the predictive character of the simulation model.

\section{Simulation models}

The fundamentals of the electro-opto-thermal simulation models are described in [1-3] and only a summary and update with respect to new models is given. The optical modes of a VCSEL are modeled by solving Maxwell's vectorial

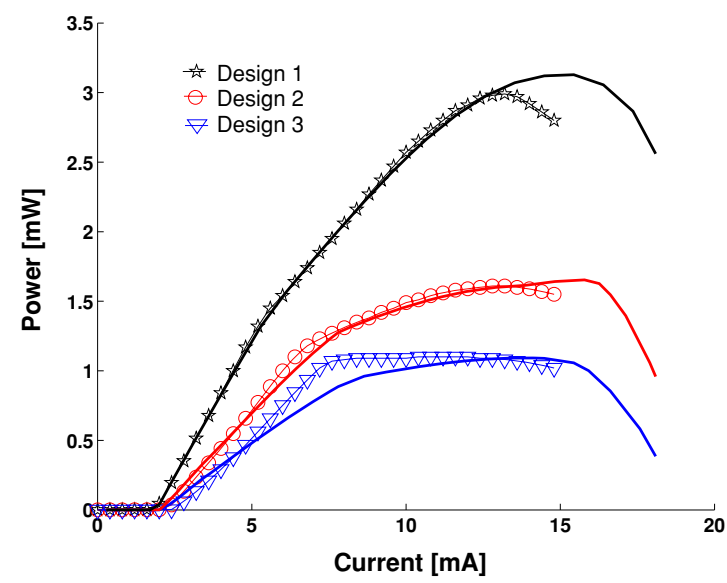

Fig. 1 Simulated (solid lines) and measured (symbols) power-current characteristics of VCSEL designs with different metal aperture radii $\left(\mathrm{Rm}_{\text {Design1 }}>\mathrm{Rm}\right.$ Design2 $\left.>\mathrm{Rm}_{\text {Design3 } 3}\right)$ at $T=303 \mathrm{~K}$. With decreasing metal aperture, the threshold currents for the fundamental and higher order modes increase due the higher absorption in the metal. Concurrently, the slope efficiency decreases since the power generated in the device is absorbed in the metal, too 


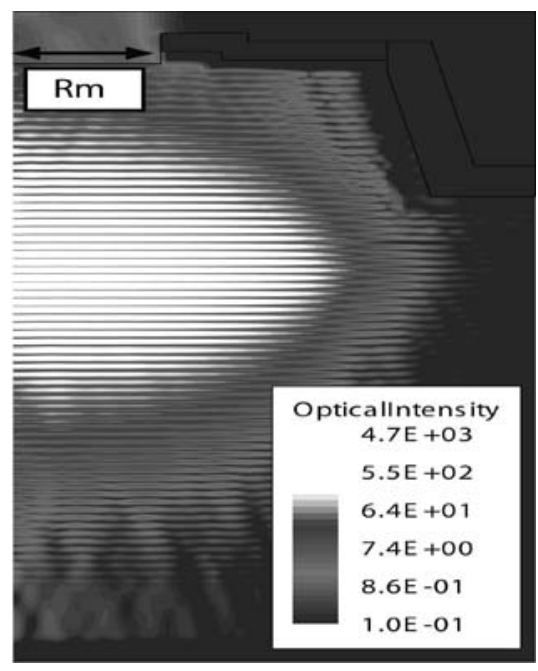

(a) $H E_{11}$ mode

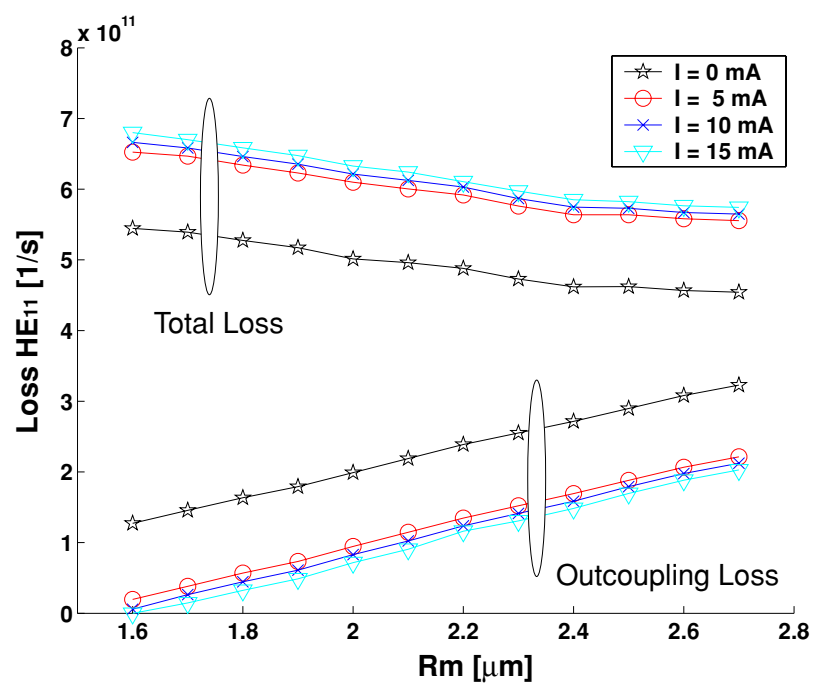

(c) Optical losses $H E_{11}$ mode

Fig. 2 Normalized optical intensity (top) and optical losses (bottom) for VCSELs with metal aperture radius $\mathrm{Rm}<2.8 \mu \mathrm{m}$. Due to absorption in the metal, the total losses for the $\mathrm{HE}_{21}$ mode are significantly higher than for the fundamental $\mathrm{HE}_{11}$ mode. This results in an improved

wave equation using a finite element method and absorbing boundary conditions. Cavity losses consist of optical outcoupling, scattering, metallic absorption, and free carrier absorption.

The electronic description comprises a Poisson equation and a drift-diffusion model for carrier transport. Thermal effects are modeled by a continuity equation for the heat flux. The interaction between carriers and the optical field is governed by the microscopic polarization based on Heisenberg's equation of motion in the second Born approximation [4]. The quantum well $(\mathrm{QW})$ bandstructure is calculated by an 8band k.p method [5]. In general, the simulator can be used to calculate the stationary, transient, small-signal, large-signal

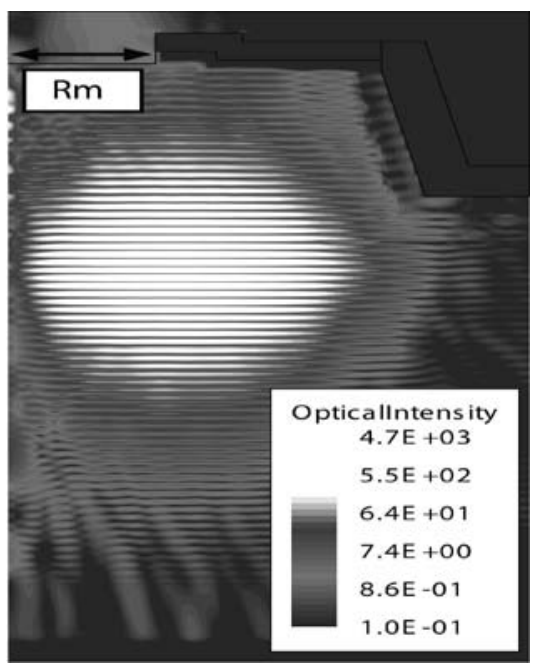

(b) $H E_{21}$ mode

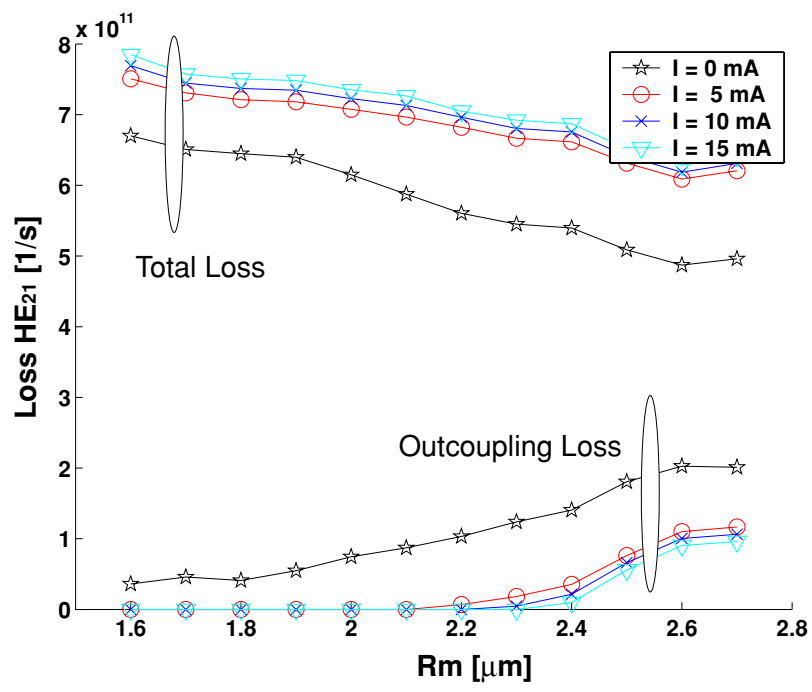

(d) Optical losses $H E_{21}$ mode

single-mode behaviour. On the other side, the optical outcoupling losses decrease with decreasing metal aperture (also caused by metal absorption) resulting in lower slope efficiency and hence, low output power

and noise characteristics of VCSEL devices in a 2-D and 3-D [6] setup.

\subsection{Single-mode VCSEL design}

Besides reliability properties, the maximum single-mode power is one of the most important performance criteria in industrial VCSEL design. A straight forward approach is the use of a narrow $(<3 \mu \mathrm{m})$ oxide aperture diameter in order to feed the carriers to the center of the $H E_{11}$ mode. However, this results in a higher differential resistance and tends to degrade the reliability. 


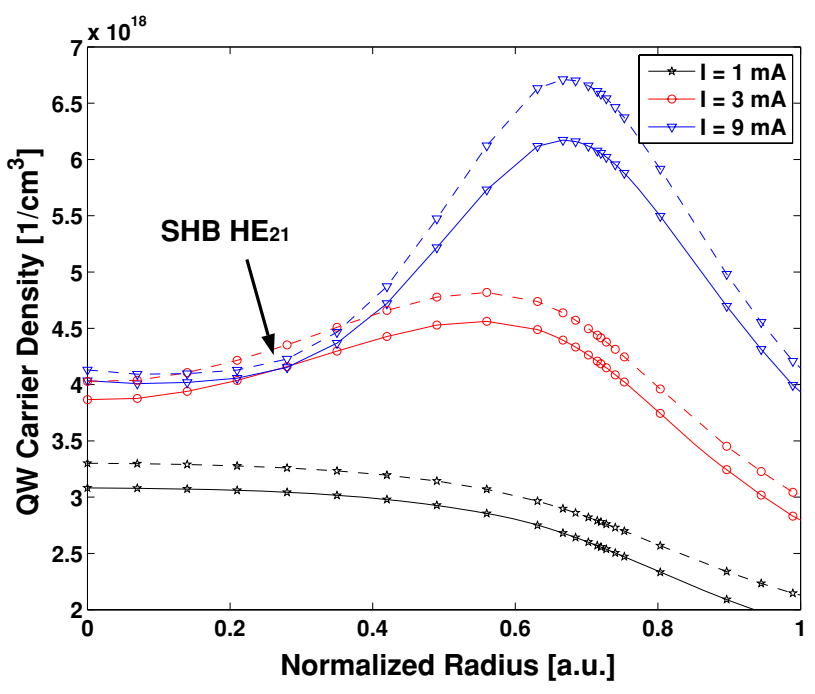

Fig. 3 QW carrier densities (solid: electrons, dashed: holes). Below threshold, the carrier concentration is almost constant up to the oxide confinement. Above threshold, spatial hole burning (SHB) occurs due to strong local stimulated recombination rates. As soon as higher order modes start to lase, multi-mode SHB is visible

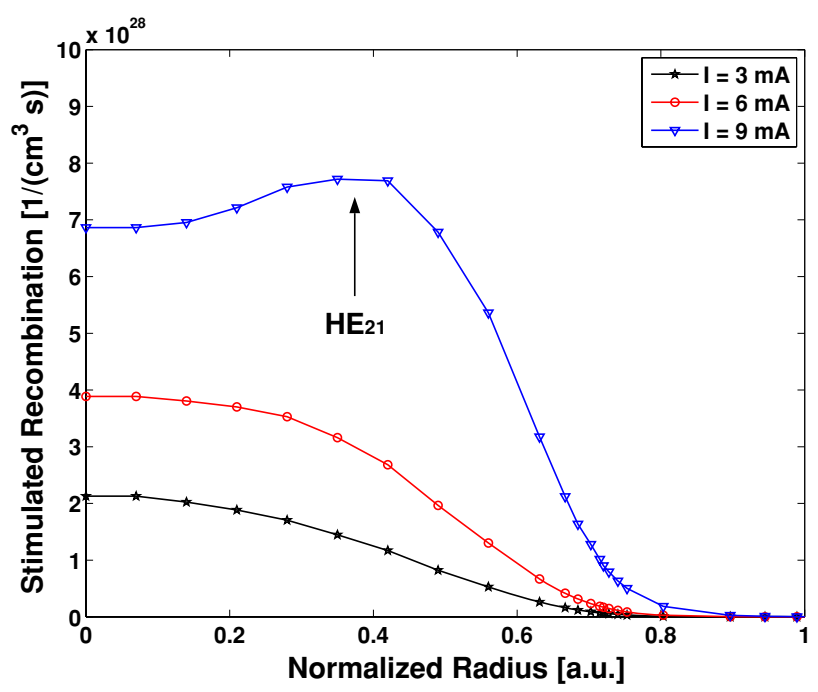

Fig. 4 Carrier recombination rates due to stimulated emission. With increasing current, the area of stimulated recombination rates increases resulting from higher material gain at larger radii with higher carrier concentration. At $9 \mathrm{~mA}$ the $H E_{21}$ mode (with threshold current $6 \mathrm{~mA}$ ) contributes already significantly to the total recombination rate

Other approaches introduce mode selective losses, either by surface reliefs, by decreasing the mesa width, or by narrowing the aperture of the contact metallization. In this work, the impact of the contact metallization aperture on the singlemode behavior of a $850 \mathrm{~nm}$ AlGaAs/GaAs VCSELs is analyzed.

\subsection{Results}

The VCSELs under investigations feature oxide diameters greater than $7 \mu \mathrm{m}$ and a different metal aperture diameters smaller than $7 \mu \mathrm{m}$. The top distributed Bragg reflector (DBR) stack is p doped and the bottom DBR stack is n doped. The device consists of a multiple QW active region made of GaAs. Devices comparable to the one used here are discussed in $[3,4,8]$. For the simulations in this work, a 2D setup has been used exploiting the rotational symmetry of the device and the basic material parameters have been taken from [7]. For single-mode investigations, the optical losses play a detrimental role. For an accurate description, the losses are calculated from the electromagnetic energy dissipation rates of the optical field vectors [3]. The contact metallization is described by a complex refractive index and free carrier absorption effects are simulated using a first order Drude model [7]

$\alpha_{\mathrm{FCA}}=\alpha_{n} n+\alpha_{p} p$

with $\alpha_{n}=5 \mathrm{e}-18 \mathrm{~cm}^{2}$ and $\alpha_{p}=11 \mathrm{e}-18 \mathrm{~cm}^{2}$, whereas $n$ and $p$ denote the electron and hole densities, respectively.

Figure 1 shows the power-current (P-I) characteristics from simulation and measurement for different metal radii $\left(\mathrm{Rm}_{\text {Design1 }}>\mathrm{Rm}_{\text {Design2 }}>\mathrm{Rm}_{\text {Design3 } 3}\right)$ at $\mathrm{T}=303 \mathrm{~K}$, which are in good agreement. The devices exhibit single-mode behaviour up to $5.5 \mathrm{~mA}$ (for design 1 ), $7 \mathrm{~mA}$ (for design 2) and $8 \mathrm{~mA}$ (for design 3 ). The maximum single-mode current can be determined either by analyzing the optical spectrum or, when the metallization is used to ensure single-mode behaviour, from the kink in the P-I characteristics due to different total and outcoupling losses of the fundamental and higher-order modes. This is completely different compared to multi-mode devices, where the total and outcoupling losses for many modes are almost equal, and no kink is visible in the P-I characteristics [8].

Figures 2(a) and (b) show the first two optical modes of a design with a top metal radius $\mathrm{Rm}<2.8 \mu \mathrm{m}$. Simulation shows that the losses increase with decreasing metal radius (see Figs. 2(c) and (d)). Concurrently, the net outcoupling losses also decrease, which results in a reduced slope efficiency. On the other hand, the threshold for the onset of the higher order mode is decreased with larger metal radius. This explains the characteristics seen in Fig. 1.

Figure 3 shows the QW carrier densities for a device with $\mathrm{Rm}=2.7 \mu \mathrm{m}$ and oxide confinement at 0.7 of the normalized radius for different bias currents (threshold current $2 \mathrm{~mA}$ and single-mode current $6 \mathrm{~mA}$ ). While the carrier density is constant below threshold, spatial hole burning (SHB) effects caused by strong local stimulated recombination rates are visible above threshold: With increasing current, the high QW 
carrier densities below the oxide caused by current crowding cannot diffuse fast enough to the center of the fundamental mode. Simultaneously, these high densities enhance the gain of the higher order modes and hence, have detrimental impact on the single-mode stability. In addition, the recombination rates of the higher order modes change the SHB pattern at very high currents (Fig. 4).

While simulation and measurement results for the threshold current, the slope efficiency and the kink in the P-I curve are in good agreement, some deviations are found for very high currents around rollover, although the trend is still correct. We suppose that this mismatch is caused by the free carrier absorption model, which may not be valid around rollover (note that the temperature increase due to self-heating at $18 \mathrm{~mA}$ is about $100 \mathrm{~K}$ in the active region and the top DBR stack).

\section{Conclusion}

In conclusion, this contribution aims to simulate $850 \mathrm{~nm}$ oxide-confined VCSELs with the aid of microscopic simulation. In particular, the single-mode behaviour is determined by a complicated interplay of electrical (current confinement), thermal (self-heating) and optical (losses) processes.
Acknowledgment The authors would like to thank Avalon Photonics for providing the characterization data, Synopsys Switzerland LLC for the simulation tools and the Swiss Commission for Technology and Innovation (CTI) for the financial support under project 6941.2 .

\section{References}

1. Synopsys Inc., Mountain View, USA, Synopsys Sentaurus Device User Manual (1995-2005)

2. Odermatt, S., Witzigmann, B.: A microscopic model for the static and dynamic lineshape of semiconductor lasers. IEEE J. Quant. Elect. 42, 538-551 (2006)

3. Streiff, M., et al.: A Comprehensive VCSEL device simulator. IEEE J. Sel. Topics Quant. Elect. 9, 879-891 (2003)

4. Witzigmann, B., et al.: Comprehensive simulation of vertical cavity surface emitting lasers: inclusion of a many-body gain model. J. Comp. Elect. 4, 7-10 (2005)

5. Odermatt, S., et al.: Bandstructure calculation using the $\mathrm{k} \cdot \mathrm{p}$ method for arbitrary potentials with open boundary conditions. J. Appl. Phys. 97, 046104 (2005)

6. Witzigmann, B., et al.: A full three-dimensional microscopic simulation for vertical-cavity surface-emitting lasers. Proc. NUSOD 5, 23-24 (2005)

7. Piprek, J.: Semiconductor Optoelectronic Devices. Academic (2003)

8. Odermatt, S., et al.: A TCAD based Yield and Reliability Analysis for VCSELs. Proc. SPIE 6132, 613206 (2006) 